\title{
Experiments with Pion-Beam
}

\author{
Verena Walbrecht* \\ Excellence Cluster Universe, Technische Universität München \\ E-mail: verena.walbrechtetum. de
}

The measurement of a pion-beam induced reactions on several nuclear targets at low energies allows to study in-medium modifications of low momentum particles. Of particular interest is the kaon nucleon which are needed to understand the interior of neutron stars and the possible existence of strangeness in such extremely dense systems.

Therefore, a pion-beam experiment has been performed with the upgraded FOPI setup in 2011, which is located at GSI Helmholtzzentrum für Schwerionenforschung. The pion-beam was impinging on three different targets $(C, C u, P b)$ at a beam momentum of $1.7 \mathrm{GeV} / \mathrm{c}$ for a comparative analysis. The upgrade of the FOPI spectrometer with a GEM-TPC (time projection chamber with GEM amplification stage) in its center improves not only tracking, but especially vertex reconstruction capabilities.

A complementary campaign is planned with the HADES detector operating also at GSI. A detailed study with simulations (GiBUU) is performed to show the feasibility of this experiment and to determine the best configurations (beam energy, target configuration) for the investigation of in-medium effects with kaons and $\Lambda$ s.

This contribution will show calibration efforts of the innovative GEM-TPC.

52 International Winter Meeting on Nuclear Physics - Bormio 2014,

27-31 January 2014

Bormio, Italy

${ }^{*}$ Speaker. 


\section{Introduction}

A Time Projection Chamber (TPC) [1] is a detector which allows a high-resolution charged particle tracking. This works even in high multiplicity enviroments like heavy ion collisions. In addition to that it is possible to identify the particles by their specific energy loss (dE/dx).

The detection volume, which is in a homogeneous electric field, is filled with gas and ends with a position-sensitive electron collection system. Furthermore, a magnetic field is parallel to the electric field, for the momentum measurements and to minimize the diffusion of the electrons coming from the ionization of the gas. Particles which fly through the detection volume produce primary ions which drift towards the anode where they are amplified and detected. Druing the amplification secondary ions are created which can drift back into the active volume where they can distort the drift field. The ion-back-flow can be reduced by using a Gas Electron Multiplier (GEM) [2] instead of the conventional MWPC as an amplification state. This is due to the suppression [3] of the GEM-foils. This supression depends strongly on the magnetic field, the gas composition and the GEM settings. That the secondary ions cause significant drift distortions, has been shown with the help of simulations[4]. A correction of these distortions with standard calibrations techniques is possible, because they are stable over timescales of ms [4], [5].

The amount of back drifting ions is small, since the reaction rate is low. So there are no significant distortions expected at a low reaction rate.

\section{The FOPI GEM-TPC}

The GEM-TPC is cylindrical and has a drift length of $725 \mathrm{~mm}$. The inner diameter is $105 \mathrm{~mm}$ and the outer diameter is $300 \mathrm{~mm}$ (Fig. 1). The field cage and the gas vessel are one entity made

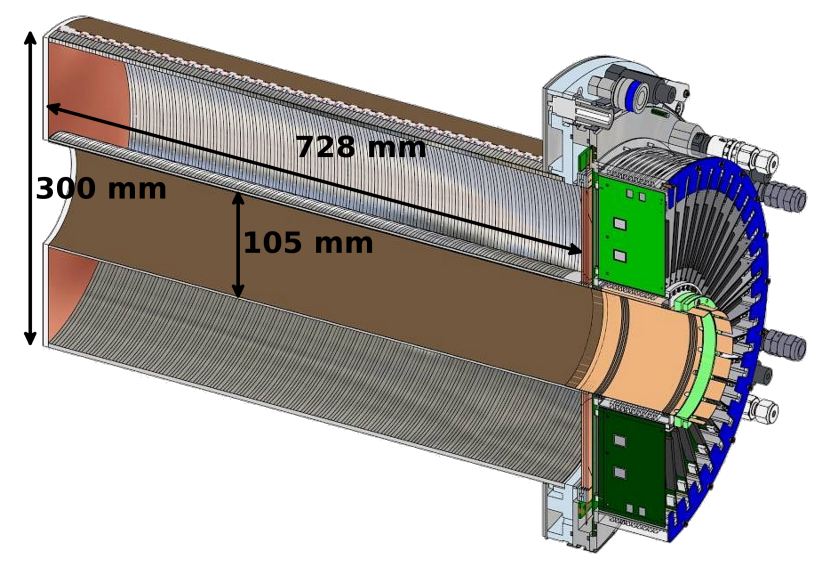

Figure 1: CAD through the FOPI GEM-TPC. The cathode is on the left side and the GEM amplification and the read out ist on the right side

out of a $\sim 4 \mathrm{~mm}$ thick sandwich structure. This structure, which is made out of ROHACELL and Kapton, allows to stay below $1 \%$ of a radiation length.

The generated electrons from primary ionization drift towards the amplification stage where they 
are amplified by a triple GEM stack. The drift field is $360 \mathrm{~V} / \mathrm{cm} .10254$ hexagonal pads are used for the readout at the pad plane. Each of them has a radius of $1.5 \mathrm{~mm}$.

The read out of the incomming signals is done with the analog sampling AFTER/T2K chip [6]. With this chip it is possible to vary the sampling frequency. Furhtermore it has an 511 cells ring buffer. For our experiment the sampling frequency was most of the time set to $15.55 \mathrm{MHz}$. The average noise for the full readout connected to the detector was $\sim 700 e^{-}$ENC [7].

\section{The experiment}

The GEM-TPC was installed inside the Central-Drift-Chamber (CDC) of the FOPI spectrometer, which is located GSI, Darmstadt (Germany). The detectors are palced in a $0.6 \mathrm{~T}$ solenoid magnetic field.

The experiment was carried out in June 2011 an the reaction was $\pi^{-}+C / C u / P b$ at an energy of $1.7 \mathrm{GeV}$. These measurements allow to study in-medium modifications of strange hadrons. For the calibration of the detector cosmic ray data were taken.

\section{Calibration}

\subsection{Comparison of the Monte Carlo (MC) simulation with a perfect drift field and the data}

For the calibration of the GEM-TPC the data of the cosmic rays are used. One parameter to compare the data and the simulation is the residual. It is the distance between a cluster of elctrons from the ionisation and the fitted track.

It turns out, that the distributions of the residuals shows local inhomogenities which spoil the momentum and vertex resolution. (Fig 2) shows the distribution of the $\mathrm{x}$ component of the residuals in the xy-plane for the data (left) in comparison to a MC simulation with a perfect drift field.
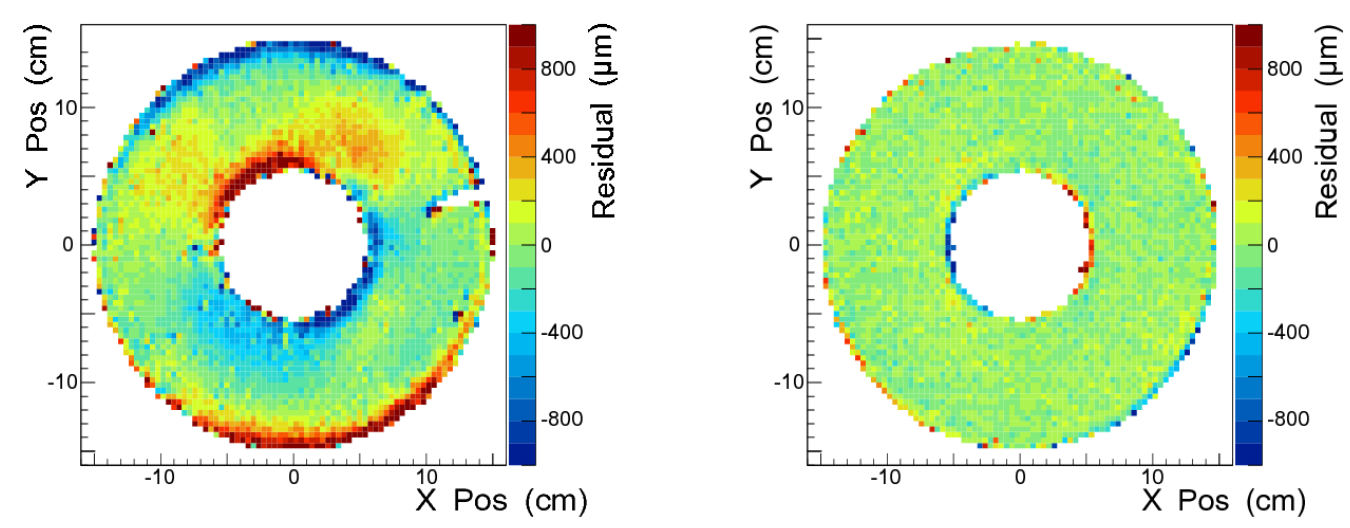

Figure 2: $\mathrm{x}$-Residuls in the xy-plane: left from the data and right from the MC simulation with a perfect drift field. 
It is obvious that this does not fit together. That suggests that there are distortions in the electric field. Therefore a drift field correction is required.

\subsection{Drift field correction}

To correct static drift field distortions it is neccessary to know the actual drift field. Therefor the field was simulated with the finite elemet methode (FEM). For this simulations the full geometry of the GEM-TPC was taken into account. This simulations includes several sources of distortions:

- Shorts of field cage strips and the cathode $(\mathrm{Z} \approx 70 \mathrm{~cm})$

- Potential at the last strip $(\mathrm{dLS})(\mathrm{Z} \approx 0.50 \mathrm{~cm})$

- Bending of the cathode $(\mathrm{Z} \approx 70 \mathrm{~cm})$

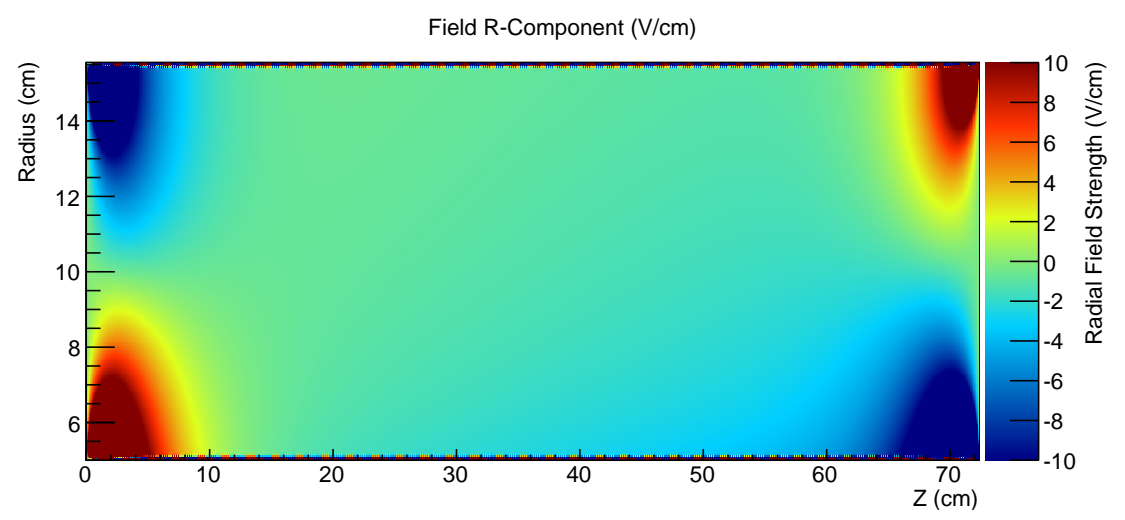

Figure 3: The resulting radial field component inside the drift volume calculated with the FEM method

The numbers of shortcut strips as well as the potential of the last strip are known from measurements while the amount of bending is unknown and has to be fixed by a systematic analysis.

The bending of the cathode was caused by fixing the inner cage. To include this distortion it is necessary to determine the bendig curve. The differential equation for the bending curve of a beam is:

$$
w(x)^{\prime \prime}=-\frac{M}{E I_{y}}
$$

where $M=F \cdot L$ the bending moment, $E$ elasticity modulus and $I_{y}=\frac{b h^{3}}{12}$ the area moment of inertia. Solving this equation with the boundary conditions for a beam fixed on one side gives:

$$
w(x)=\frac{F \cdot l^{3}}{6 \cdot E \cdot I_{y}}\left(\frac{x}{l}\right)^{2}\left[3-\frac{x}{l}\right]
$$

the resulting bending curve was implemented stepwise with a stepsize of $1 \mathrm{~mm}$ since a continous implementation would result in the need of a very fine mesh and therefor increase the calculation time significantly. While implementing the bending curve the boundary effects of the fieldcage walls were also optimized by improving the implementation of the potentials of the field strips.

With the knowledge of the effective drift field and the magnetic field it is possible to calculate the 
drift path of the electrons in the distorted field by solving thee the Langevin equation numerically. Thereby a map of drift distotions as a function of the starting point of the drift can be created[4]. The equation of motion is given by:

$$
m \frac{\mathrm{d}}{\mathrm{d} t} \mathbf{u}^{-}=e \mathbf{E}+e\left[\mathbf{u}^{-} \times \mathbf{B}\right]-K \mathbf{u}^{-}
$$

where $\mathbf{u}^{-}$is the macroscopic electron drift velocity, $e$ the electron charge and $K=e / \mu^{-}$a constant friction term.

\subsection{Comparision of the MC simulation with distored field and the data}

The MC simulation with the distorted drift field fits much better to the data. (Fig.4) shows the $\mathrm{x}$ component of the residulas in the xy-plane of the data in compare to the MC simulation taking the distorted field into account.
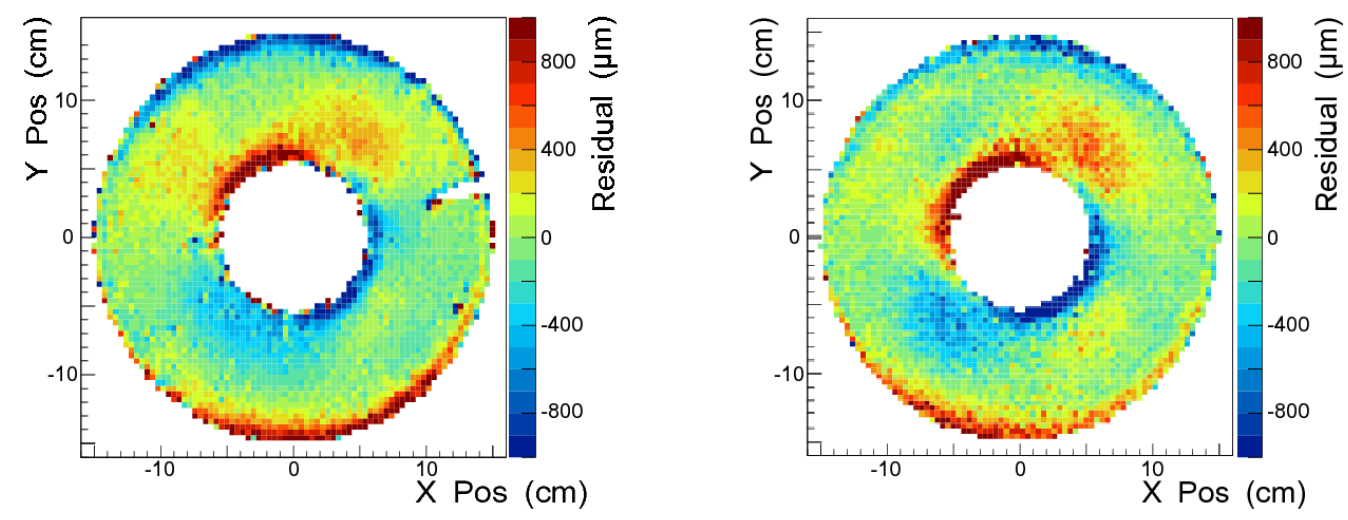

Figure 4: $x$-Residuls in the xy-plane: left from the data and right from the MC simulation with the distorted field.

To look how efficent the simualtions are we calculate the relative difference (RD) of each bin, which is given by:

$$
R D=\frac{\operatorname{Res}_{\text {Data }}-\operatorname{Res}_{\text {Sim }}}{\left|\operatorname{Res}_{\text {Sim }}\right|}
$$

The distribution of the relative difference for the comparison between the data and the simulations without a distorted field has a mean of -0.80 and a $\sigma$ of 9.65 . When taking the distortions into account these values improve to 0.006 for the mean and 2.28 for the standard deviation.

\section{Outlook}

The parameters for the severeal distortions are not fixed. That is why a optimization of the variable parameters (dLs and the bending of the curve) is in progress at the moment to find the best accordance with the data and the simulation. After that it is possible to correct for the drift distortions. 


\section{References}

[1] D. R. Nygren, et al., Phys. Today 31N10 (1978) 4.

[2] F. Sauli, et al., Instr. Meth. A 386 (1997) 531.

[3] F. Sauli, et al.,IEEE Trans. Nucl. Sci. 50 (2003) 803.

[4] F.V. Boehmer, et al., Nuck. Instr. Meth. A 719 (2013) 101-108.

[5] ALICE Collaboration, L. Musa, et al., Letter of intent for the upgrade of the alice experiment, Tech. Rep. CERN-LHCC-201-012. LHCC-I-022, CERN, Geneva (Aug 2012)

[6] P. Baron, et al., IEEE Trans. Nucl. Sci. 55 (2008) 1744

[7] S. Dørheim, 2012 JINST 7 C03011 